Journal of Advanced College of Engineering and Management, Vol. 6, 2021

\title{
ARTIFICIAL NEURAL NETWORK BASED APPROACH FOR VOLTAGE STABILITY ANALYSIS OF FOR SUSTAINED OPERATION OF POWER SYSTEM
}

\author{
Yuba Raj Adhikari ${ }^{1}$, Rishi Kr. Barnawal ${ }^{2}$ \\ ${ }^{1}$ Department of Electrical Engineering, Pulchowk Campus, IOETU, \\ ${ }^{2}$ Nepal Electricity Authority
}

\begin{abstract}
For reliable and secure power system, the stability analysis is recognized as an important problem. Voltage stability is the capacity of a power system to maintain steady acceptable voltages at all buses in the system. Voltage stability index (VSI) evaluation for a situation of power system can act as an accurate and fast indicator of the proximity of the system to voltage instability. Recently there has been considerable interest in intelligent methods based on artificial neural network (ANN), fuzzy logic and genetic algorithm to voltage stability assessment problem. ANN, with the ability to provide non-linear input/output mapping, parallel processing, learning and generalization have the potential to make them ideally suited for estimating VSI's of a power system without solving the governing power system equations. This paper is to purpose an alternative method using ANN for finding the closeness of system operating point to voltage collapse that would be claimed to have better computational speed, accuracy, efficiency and reliability. Voltage Stability Analysis (VSA) using VSI is performed for different alternate loading strategies of power network building ANN models for every different scenario. The outcome found working satisfactorily in analyzing voltage stability problem, basically in ranking the network buses according vulnerability order.
\end{abstract}

Keywords: Power system stability, Voltage stability index, Artificial Neural Network, Error-back propagation, weak bus.

\section{Background}

Power system stability has been recognized as an important problem for secure system operation since several decades. Side by side, voltage stability assessment and control are not considered as any new issue [1], but they have been attaining special attentions to maintain the stability of the power networks in order to avoid recurrence of major blackouts as experienced by the particular countries. The power system can be classified in the voltage stability region if it can maintain steady acceptable voltages at all buses in the system under normal operating conditions and after being subjected to a disturbance [2]. In order to be reliable, the power system must be stable at most of the time.

Methods available for voltage stability studies can be broadly categorized into static and dynamic [2]. Static methods assume a steady state model or a linearized dynamic model to investigate the state of the equilibrium point of a specified operating condition of the power system. For dynamic methods, the solution of governing equations is obtained in time domain, and the study period is in the order of several minutes. Dynamic simulations are time consuming and complex for assessment of system stability. The static approach addresses the questions: How close the system is to voltage instability? It is concerned with the determination of the proximity of the system state to the voltage instability boundary. The dynamic approach addresses the question: How and why does instability occur? It is concerned with the mechanism of Voltage Instability [3]. The first question is of vital importance to utilities in order to operate their system with maximum security and reliability. There is a need for an accurate and fast indicator of the proximity of the system to voltage instability. For static approach, many methods have been proposed to determine the proximity to voltage instability. One of the methods among various methods is to use static voltage stability indices which quantify the proximity of system operating point to voltage collapse [4]. They give early detection of a possible voltage collapse of the power system.

There has been considerable studies in intelligent methods based on artificial neural network (ANN), fuzzy logic and genetic algorithm to voltage stability assessment problem because of various 
advantages offered by them including their high computational rates and so on[5][6][7][12a].[3a] For an instance, ANN's are emerging Artificial intelligence tool for on-line applications. An ANN is a massively parallel information-processing system. It can perform non-linear computations in a short duration [new 1]. Due to various advantages offered by Artificial Neural Network (ANN), there is increasing trend to use it in accessing voltage stability of system. ANN, with the ability to provide nonlinear input/output mapping, parallel processing, learning and generalization have the potential to make them ideally suited for estimating VSI's of a power system without solving the governing power system equations [8].

The objective of the paper is to purpose an alternative method using ANN for finding the closeness of system operating point to voltage collapse that would be claimed to have better computational speed, accuracy, efficiency and reliability. The scope of the study is just not limited to find the proximity of system to voltage collapse. The same method can be equally useful to observe the impact of line outage in the system whereby contingency analysis is performed since the procedure for contingency analysis is almost similar to the one in voltage stability analysis (VSA).

The coming chapters envisage the overview of the voltage stability. Additionally, ANN fundamental as well as its superiority for handling a nonlinear system analyzing is briefly described. There after aANN based methodology is presented in following chapters. There after results are analyzed and finally some conclusions are presented.

\section{Voltage Stability Analysis: Concepts and Evaluation Approach}

A vital element i.e. voltage stability, among various factors of power system stability, is the capacity of a power system to maintain steady acceptable voltages at all buses in the system at normal operating conditions and after being subjected to a disturbance.

Small-disturbance (or small-signal) voltage stability is concerned with a system's ability to control voltages following small perturbations, such as gradual changes in load. This form of stability can be effectively studied with steady-state approaches that use linearization of the system dynamic equations at a given operating point. Voltage stability analysis can be performed in a power system by evaluating the voltage stability index (VSI). One among various methods is to use static voltage stability indices which quantify the proximity of system operating point to voltage collapse. There is no standard definition for voltage stability index but a simple voltage stability index (VSI) derived from fundamental principle can be utilized for the voltage stability studies.

Solving and rearranging according to [1] in a two-bus system power network, the condition obtained is presented as in equation as below

$$
\frac{4 Z^{2} Q_{2} X}{V_{1}^{2} \cdot(R \cdot \operatorname{Sin} \delta+X \cdot \operatorname{Cos} \delta)^{2}} \leq 1.0
$$

Generalizing the above equation (2.1) by taking ' $i$ ' as the sending end bus and ' $\mathrm{j}$ ' as the receiving end bus, the voltage stability index (VSI) is defined as,

$$
V S I_{i j}=\frac{4 Z^{2} Q_{j} X}{V_{i}^{2} \cdot(R \cdot \operatorname{Sin} \delta+X \cdot \operatorname{Cos} \delta)^{2}}
$$

Thus, index VSI must be less than unity for a system to be voltage stable. The value of VSI index can indicate voltage stability condition of the system. VSI value close to 1.0 indicates that the particular line is close to its instability point which may lead to voltage collapse in the entire system. To maintain a 
secure condition the value of VSI should be maintained well below 1.0. The above relation of VSI is true for power system with any number of buses.

\section{Artificial Neural Network: Fundamental and relevance to Voltage Stability}

Artificial neural network (ANN) is computational analogs of the basic biological components of a brain - neurons, synapses and dendrites. ANNs consist of many simple computational elements (summing units - neurons - and weighted connections - weights) that work together in parallel and in series. Neural network begin in a random state and "learn" using repeated processing of a training set that is a set of inputs with target outputs. The fundamental processing unit of neural network is the neuron i.e. the interconnection of these neurons form a Neural Network. Basically, a neuron receives inputs from other sources, combines them in some way, performs an operation which is generally non-linear and then gives the final output which might be the final output or input to other neurons.

Once a network has been structured for a particular application, the network is ready to be trained. The hidden layer in the ANN is basically to introduce the non-linearity. To start this process the initial weights are chosen at random. Then, the training or learning process of neurons begins. There should be some proper learning algorithm to train these networks [9]. ANN models are found being implemented to predict the performance indices for unseen network conditions and rank them in vulnerability order based on performance indices for security assessment [10] [11].

\section{Methodology}

ANN based VSI estimation method overview is depicted in flow chart below.

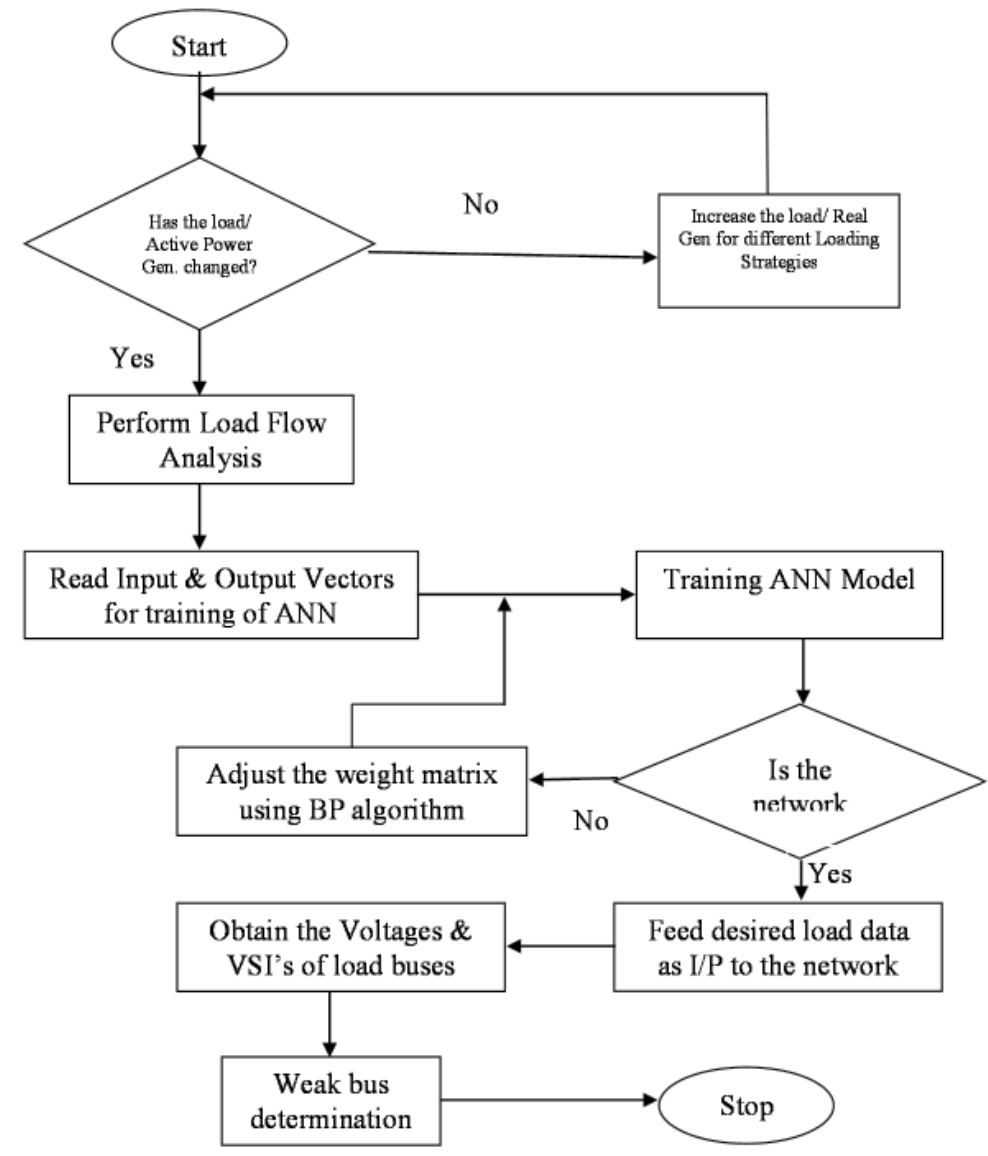

Fig 4.1 Flow Chart for voltage stability studies by ANN 
The feed-forward network with three layers of neurons, one input layer, one output layer and a single hidden layer as shown in the figure 4.2 below is chosen for the purpose. The proposed architecture will be as shown below.

Input Nodes $\geq 2 *$ Number of Load Buses

Output Nodes $=2 *$ Number of Load Buses

Layers $=1$ input layer, 1 output layer and 1 hidden layer.

The training of the proposed network is done in offline mode. The training data for voltage stability probiem is obtained from the solution of Load-Flow by Matpower Version 3.0.0, a Matlab Power System Simulation Package developed by Power System Engineering Research Center (PSERC) [13]. It uses Newton's method for the solution of the Load-Flow.

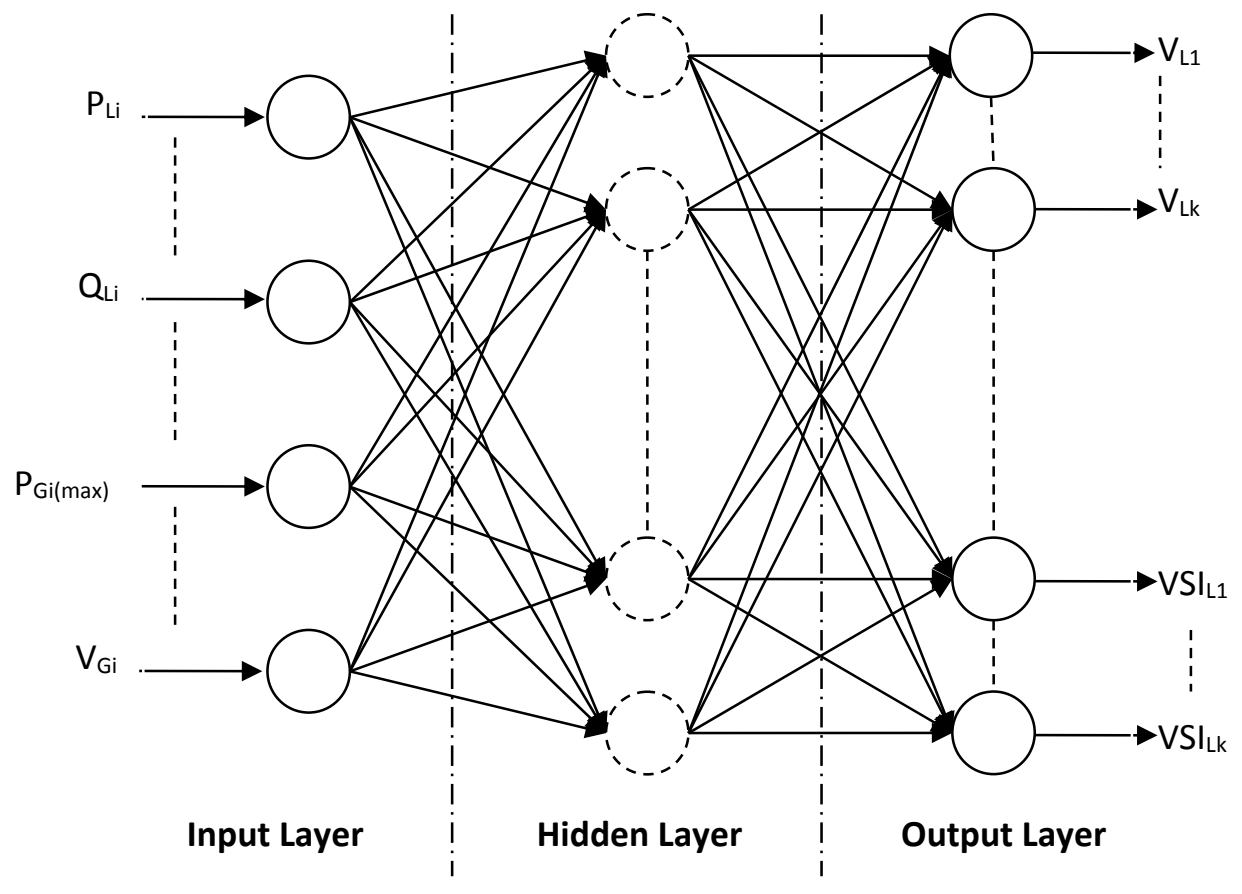

Fig 4.2 Feed-Forward Network of ANN

The solution obtained from the neural network will be compared and validated by comparison with known input-output pattern. The validation pattern is also obtained from the same simulator.

When the network is trained satisfactorily it is used for online application as follows:

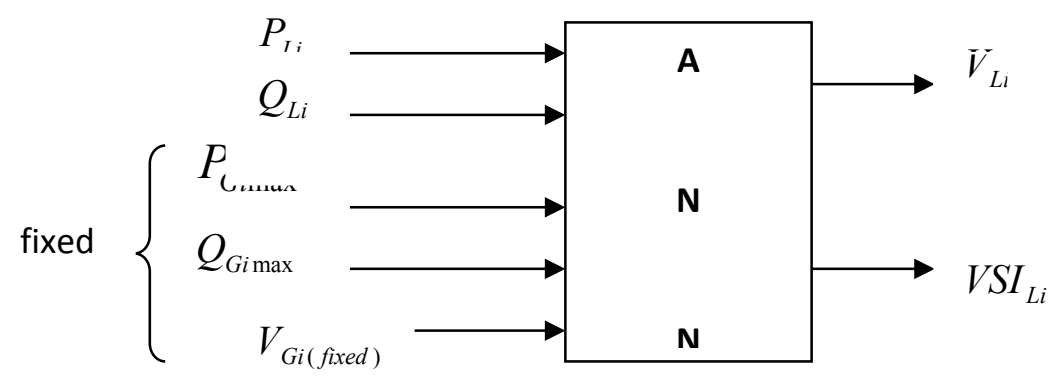

Fig 4.3 Block Diagram of ANN 
Active power and Reactive power at load buses, PLi and QLi are input to the neural network and the voltages, VLi and VSILi's of all the load buses are output of the neural network. For this study, the real and reactive power limits of the generator have been set to the maximum values. The generator voltages remain unchanged, amplitude and phase wise.

For the 6-bus test system [13], the output to the neural network has been taken, the voltages and VSI's at load buses 4,5 and 6. Voltages and VSI's are computed after load-flow simulation using MATPOWER 3.0.0 and equation (2.2).

\section{Results and Discussion}

Voltage Stability Analysis (VSA) using VSI is performed for different alternate loading strategies to obtain input/output data set to train ANN. Following four cases of loading strategies is studied.

CASE 1: Increasing only reactive power of all load nodes by constant loading factor (LF) of 0.005 .

CASE 2: Increasing real and reactive power of all nodes by constant loading factor (LF) of 0.005 .

CASE 3: Increasing real and reactive power of all loads and only the active power of the generators (except slack bus gen.) by constant loading factor (LF) of 0.005 .

CASE 4: Increasing real and reactive power of each load bus by different loading factor (i.e. bus 4 by LF 0.005 , bus 5 by LF 0.01 and bus 6 by LF 0.02).

The network is trained based upon different combination of activation functions viz. purelin, logsig, tansig and training functions viz. traingd, traingdm, traingda, traingdx and trainlm to achieve the optimum combination depending upon the training period and performance error (MSE). Based upon training period and performance error (MSE), the combination of activation functions tansig - tansig purelin with training function trainlm was proved to be most suitable for the data set used for study.

Fig. 5.3, 5.4 and 5.5 shows the performance of the ANN in predicting the highest VSI as well as decline in the voltage associated with respective bus. No significant difference between the actual and Neural Network results is observed.

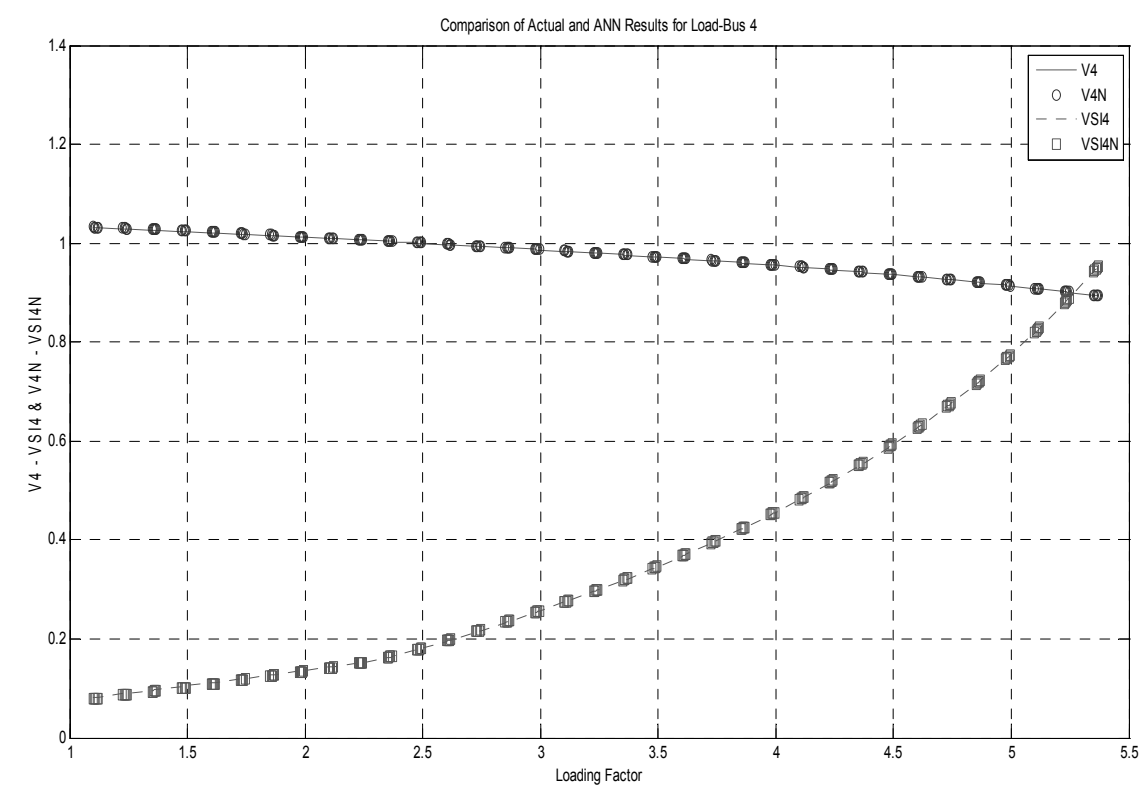

Case 2: Comparison of Actual and ANN Results for Load-Bus 4

Fig. 5.3

jacem, vol.6, 2021 Artificial Neural Network Based Approach For Voltage Stability Analysis Of For Sustained Operation Of Power System 


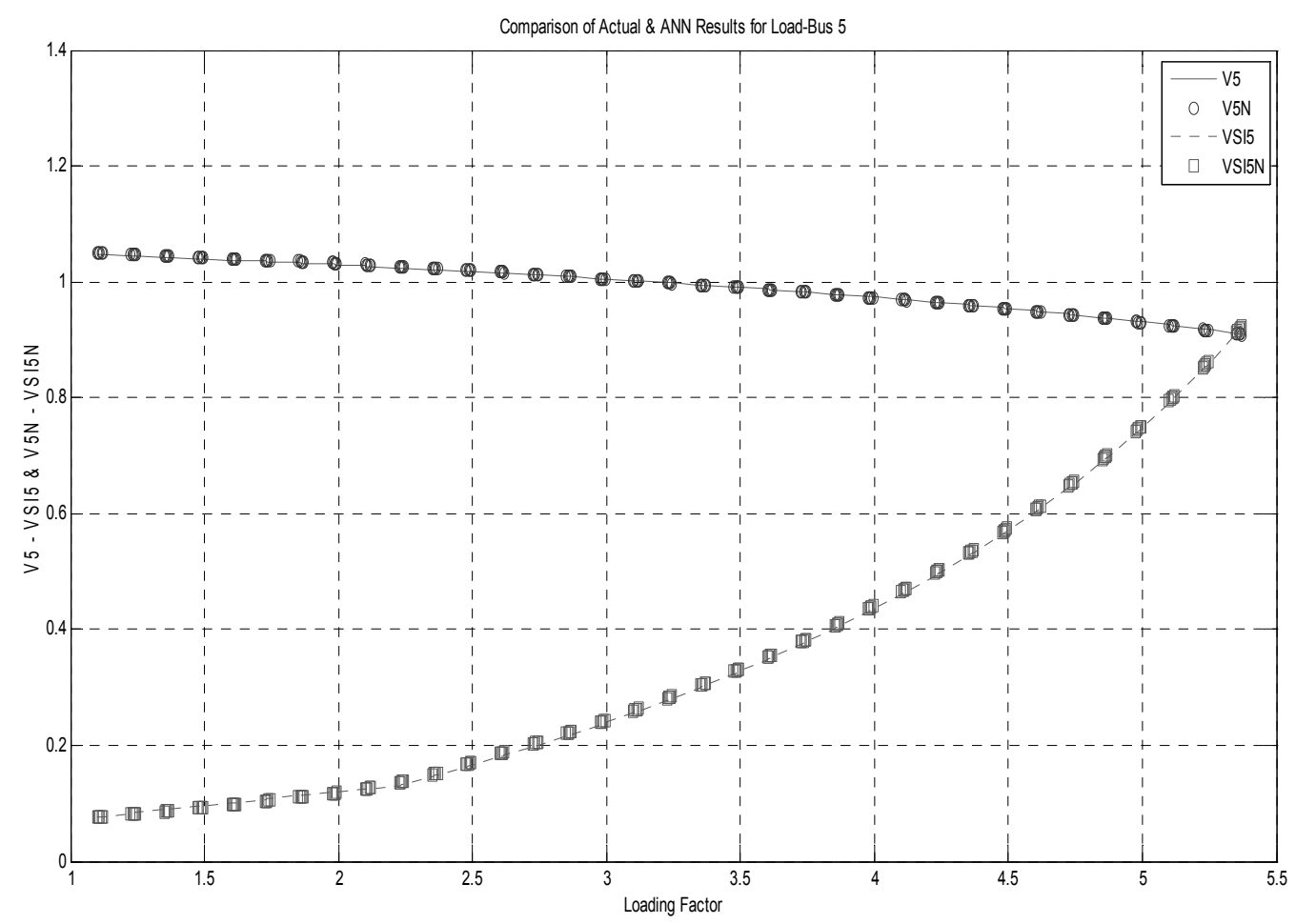

Fig. 5.4 Case 2: Comparison of Actual and ANN Results for Load-Bus 5

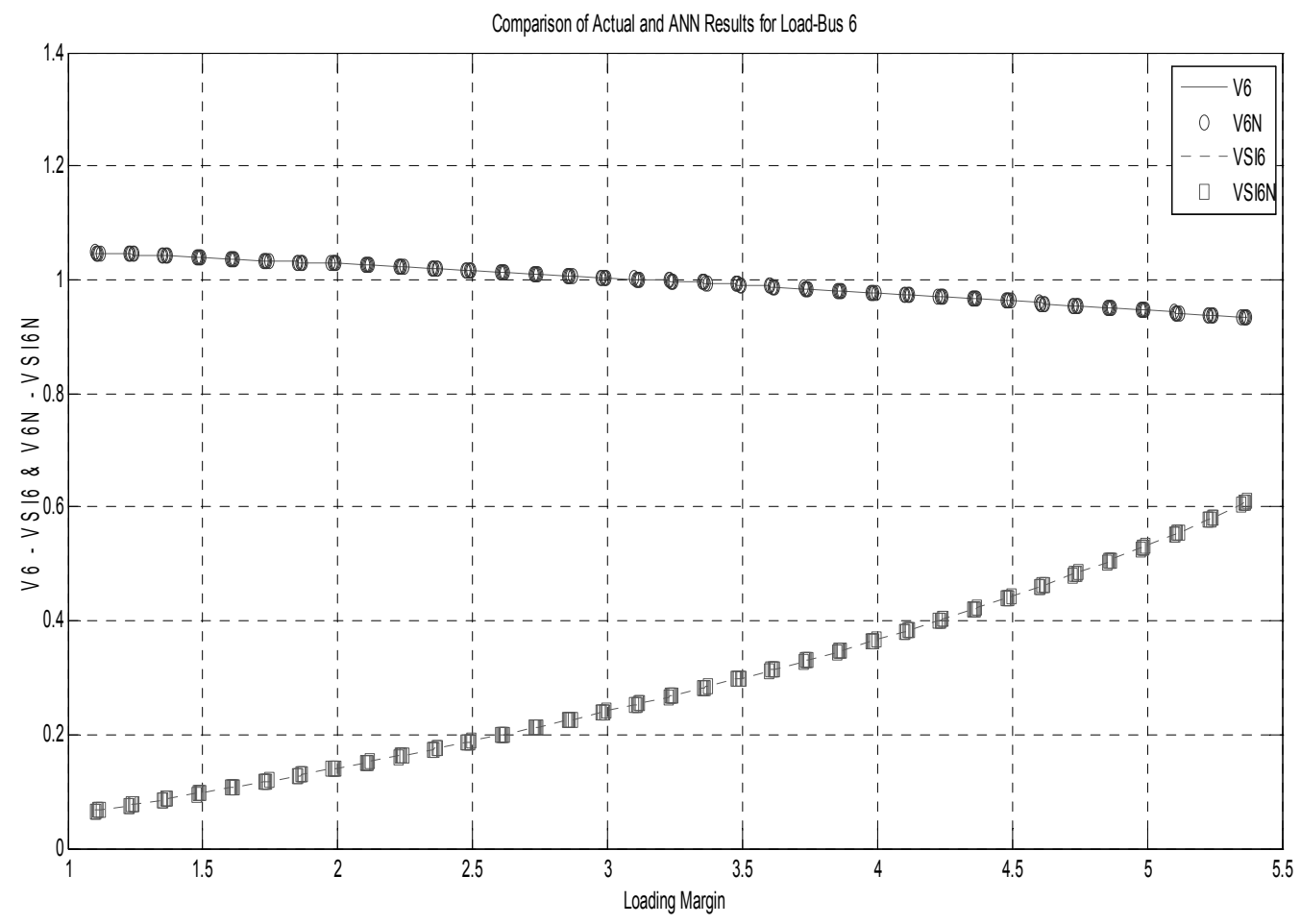

Stop Training

Fig. 5.5 Case 2: Comparison of Actual and ANN Results for Load-Bus 6 
Table 5.5 Results Comparison of different Loading Conditions to find VSIs

\begin{tabular}{|c|c|c|c|c|c|c|}
\hline S.No. & & CASE 1 & CASE 2 & CASE 3 & CASE 4 & Remarks \\
\hline 1 & Voltage of Load Bus 4 (pu) & 0.8882 & 0.8922 & 0.8499 & 0.9750 & \\
\hline 2 & Voltage of Load Bus 5 (pu) & 0.9658 & 0.9086 & 0.8880 & 0.9285 & \\
\hline 3 & Voltage of Load Bus 6 (pu) & 0.9111 & 0.9315 & 0.8994 & 0.8769 & \\
\hline 4 & VSI Value at Bus 4 & 0.9880 & 0.9684 & 0.9785 & 0.5701 & \\
\hline 5 & VSI Value at Bus 5 & 0.4414 & 0.9354 & 0.6651 & 0.9681 & \\
\hline 6 & VSI Value at Bus 6 & 0.8213 & 0.6153 & 0.7783 & 0.9699 & \\
\hline 7 & $\begin{array}{l}\text { Ranking of Buses with Decreasing } \\
\text { Order of Weakness }\end{array}$ & $\begin{array}{l}\text { Bus4 - } \\
\text { Bus6 - } \\
\text { Bus5 }\end{array}$ & $\begin{array}{l}\text { Bus4 - } \\
\text { Bus5 - } \\
\text { Bus6 }\end{array}$ & $\begin{array}{l}\text { Bus4 - } \\
\text { Bus6 - } \\
\text { Bus5 }\end{array}$ & $\begin{array}{l}\text { Bus6 - } \\
\text { Bus5 - } \\
\text { Bus4 }\end{array}$ & \\
\hline 8 & $\begin{array}{l}\text { Maximum Loading Factor that can be } \\
\text { increased before reaching Voltage } \\
\text { Instability }\end{array}$ & 10.995 & 5.395 & 6.745 & 2.645 & \\
\hline 9 & $\begin{array}{l}\text { Maximum Active Power Generation for } \\
\text { Gen. Bus 2, } \mathrm{P}_{\mathrm{G} 2}(\mathrm{MW})\end{array}$ & 50.000 & 50.000 & 337.250 & 50.000 & \\
\hline 10 & $\begin{array}{l}\text { Maximum Active Power Generation for } \\
\text { Gen. Bus } 3, P_{\mathrm{G} 3}(\mathrm{MW})\end{array}$ & 60.000 & 60.000 & 404.700 & 60.000 & \\
\hline 11 & $\begin{array}{l}\text { Maximum Active Power Loading for } \\
\text { Bus 4, } \mathrm{P}_{\mathrm{L} 4}(\mathrm{MW})\end{array}$ & 40.000 & 215.800 & 269.800 & 105.800 & \\
\hline 12 & $\begin{array}{l}\text { Maximum Reactive Power Loading for } \\
\text { Bus 4, QL4 (MVAR) }\end{array}$ & 219.900 & 107.900 & 134.900 & 52.900 & \\
\hline 13 & $\begin{array}{l}\text { Maximum Active Power Loading for } \\
\text { Bus 5, } \mathrm{P}_{\mathrm{L} 5} \text { (MW) }\end{array}$ & 40.000 & 215.800 & 269.800 & 171.600 & \\
\hline 14 & $\begin{array}{l}\text { Maximum Reactive Power Loading for } \\
\text { Bus 5, Q Q } 5 \text { (MVAR) }\end{array}$ & 54.975 & 26.970 & 33.725 & 21.450 & \\
\hline 15 & $\begin{array}{l}\text { Maximum Active Power Loading for } \\
\text { Bus 6, } \mathrm{P}_{\mathrm{L} 6} \text { (MW) }\end{array}$ & 40.000 & 215.800 & 269.800 & 303.200 & \\
\hline 16 & $\begin{array}{l}\text { Maximum Reactive Power Loading for } \\
\text { Bus 6, Q Q } 6 \text { (MVAR) }\end{array}$ & 219.900 & 107.900 & 134.900 & 151.600 & \\
\hline
\end{tabular}

In each case, the loading factor (LF) is increased near up to voltage instability point (i.e. VSI approaching to 1.0 as shown in Table 5.5. For case 1, LF can be increased up to 10.995 (highest among all cases), while for case 4 , it is least i.e. 2.645. In case 2, where both real/reactive powers of all load nodes were increased, the LF could only be increased up to 5.395. As real power generation were also increased along with loads in case 3, the LF can be increased up to 6.745. Depending upon highest VSI values, ranking of buses with decreasing order of weaknesses of voltage stability is shown in Table 5.5. Moreover, maximum loadability of load nodes can be found out by the same work as shown in the same table stated above. 


\section{Conclusion}

The research purposes ANN based approach in power system for early prediction of the proximity to voltage collapse. The method was tested in a 6-bus test system [13]. The training and testing data set were obtained by applying different alternate loading strategies using Newton's load-flow method from matpower package, MATPOWER 3.0.0, a Matlab Power System Simulation Package developed by Power System Engineering Research Center, (PSERC).

It was found that instability was quickly achieved in situation where both real/reactive power of all load nodes were increased (Case 2) as compared with situation where only reactive power of all load nodes were increased (Case 1). From voltage stability point of view, the worst case was the situation where real/reactive powers of each load node were increased by different loading factor (Case 4). In this situation the maximum loadability of the system was also less. The voltage stability analysis using VSI provides information of the weak bus in the system.

The VSI is an indicative instrument in evaluating the closeness of point of voltage instability and can be used as the measuring instrument, since it has a very simple structure, can be handled easily and can be extended to a multi-node system. The results presented indicate the possibility of using the technique for on-line voltage stability prediction, especially for practical power systems in comparison with real simulation where large computation time is required. 


\section{References}

1. J. Hongjie, Y. Xiaodan and Y. Yixin. "An improved voltage stability index and its application". Electrical Power and Energy System 27, 2005.

2. P. Kundur, "Power System Stability and Control", Second Reprint, Tata McGraw-Hill Publishing Co. Ltd., New Delhi, 2006.

3. C. Subramani, S.S. Dash, M. Jagadeeshkumar, "Voltage Stability based Collapse Prediction and Weak Cluster Identification", Int'l Journal of Electrical \& Power Engg. 3(2): pp 124-128, 2009.

4. J. Modarresi, E.Gholipour\& A. Khodabakhshian. "A comprehensive review of the voltage stability indices". enewable and Sustainable Energy Reviews, Volume -63, 2016.

5. P.K. Satpathy, D. Das, P.B.D. Gupta, "Static Voltage Stability Analysis using Fuzzy Set Approach", IE(I) Journal-EL, Vol.85, pp 49-54, June 2004.

6. M. Nizam, A. Mohamed, M. Al-Dabbagh, A. Hussain, "Using Support Vector Machine for Prediction Dynamic Voltage Collapse in an Actual Power System", PWASET, Vol.31, pp 711716, July 2008.

7. S.I. Suliman, T.K.A. Rahman, I. Musirin, "Artificial Immune-based for Voltage Stability Prediction in Power System", AIML Int'l Conference, Sharm El Sheikh, Egypt, pp 41-46, June 2006.

8. X. Gu, C.A. Canizares, "Fast Prediction of Loadability Margins using Neural Networks to Approximate Security Boundaries of Power Systems", IET Generation, Transmission \& Distribution Publication, pp 1-16, Dec. 2006.

9. S. Haykin, "Neural Networks", Second Edition, Pearson Education India, 2001.

10. P. Shekhar and S. Mohanty. "An online power system static security assessment module using multi-layer perceptron and radial basis function network". Electrical power and energy systems, vol. 76, pp. 165-173, 2016.[2a]

11. H. Shah and K. Verma,. "Voltage stability monitoring by different ANN architectures using PCA based feature selection". IEEE 7th Power India International Conference, 2016.

12. H. Demuth, M. Beak, M. Hagan, "Neural Network ToolboxTM 6", User's Guide, http://www.mathworks.com, 2008.

13. R.D. Zimmerman, C.E. Murillo-Sanchez, D. Gan, "MATPOWER VERSION 3.0.0", a Matlab Power System Simulation Package, PSERC, Feb. 2005, http://www.pserc.cornell.edu/matpower 